\title{
Are We as Attentive to Method as We Should Be?
}

\author{
Birgitta HöiJer
}

Interest in methodological issues in the field of mass communication and media studies is experiencing something of a revival. We find evidence of the trend in publishing, as well as the themes of conferences and postgraduate courses. Looking back we can see a longstanding tension surrounding methodology in our field, which is a something of a hybrid of influences from the social sciences and the humanities.

In the social sciences issues of methodology have traditionally been quite central - too central, some critics say, and point out that certain dominant methods have tended to steer the focus of enquiry rather than the research question, as should be the case. A sort of positivist methodological fundamentalism has prevailed, a preoccupation with method, to which both the content of the research and issues of relevance have been subordinated. Say critics.

Within the humanities, by contrast, attention to methodology has been slight and not at all central. That is, relative to the social scientific commandment to make one's methodology explicit that we find in textbooks, postgraduate courses and discussions of method in dissertations, articles and research reports. Method is a non-concept within classical studies of the Arts and methodological questions are usually quite peripheral. Fetveit (2000) writes quite frankly that methodology is simply less relevant in the Humanities. As a consequence, courses and books devoted to choice of method have been scarce, as has discussion of methodology in dissertations. The notion that in the humanities it is impossible to separate theory from method, or theory from empirical analysis, is - or has been widespread. They are so closely integrated that there is no need to differentiate a part called methodology and subject it to judgement or reflection. This may be the root of the antagonism toward methodological concerns that imbues a good share of research in the humanities to date.

Obviously, the comparison is rendered in very broad strokes. Still, it is useful as a backdrop to different basic perspectives on issues of methodology that we encounter in social science and Arts studies of media and communication. The field is also highly multidisciplinary, with influences from Sociology, Political Science, Psychology, Ethnology, History, Literary Criticism, Linguistics and Film Studies. This makes for a miscellaneous assortment of methods to take into account. Is such an undertaking even possible?

In my view, we have lost sight of several important aspects of the question of methods and methodology. To some extent, it may be a consequence of the great variety of methods being used in our field, but it may also be symptomatic of more sinister tendencies relating to choice of methods in scientific inquiry more generally. I shall return to this. In the following, "methodology" is used to designate a meta-perspective on methods, e.g., treatments of methods in theoretical, epistemological and ontological perspectives. "Methods" denotes more scientific procedure and concrete approaches - in short, how we go about it. The two levels are equally important, and it is also important to discuss how they relate to each other.

A disclaimer is called for here. The criticism I put forward in the following does not apply to the whole of research in our field; rather, I am referring to certain tendencies that, in my view, threaten to undermine our research - and others'. The criticism is not confined to research in Sweden and the Nordic region, nor does it apply uniquely to studies of media and mass communication. 


\section{Methodological Stringency, A Thing of the Past?}

Nowadays, in reports of quantitative survey research we frequently find frequencies of non-response as high as $40 \%$, sometimes as high as $50 \%$. Data that previously would have landed in the rubbish bin due to excessive non-response are reported, sometimes with no discussion whatsoever of the composition of the non-response or how it may have influenced the findings. Twenty to thirty years ago, when I worked at the Audience and Programme Research Department of the Swedish Broadcasting Corporation, the limit of tolerance was $20 \%$ non-response, $10 \%$ in the case of telephone interviews with random samples of the population. The limit was seldom reached; non-response was generally considerably lower. Clearly, people's willingness to take part in telephone surveys is not what it used to be. The ubiquity of market research, an eroded respect for authority and more stressful lives all contribute, as does the language barrier, due to recent immigration. As a consequence, sizeable frequencies of non-response are something we must accept. They are, however, seldom recognized as a problem. A penetration of how non-response may have influenced one,s results should be part of any serious study.

Another common fault is that the validity of questions is seldom scrutinized. Yet another: only the most rudimentary statistical analyses are applied. In some cases no multivariate analysis has been applied to control for interaction between variables.

In qualitative studies convenient samples are now accepted in place of stringent strategic sampling. These often masquerade under the alias of "snowballing": the researcher vacuums friends, and associates' networks for appropriate informants. Interviewing is not seen to require any particular skill or methodological training. Interviewing is in fact a sophisticated undertaking that requires both theoretical sensibilities and considerable experience, yet ordinary social competence is accepted in place of professional skill. When it comes to analysis of interview material, authors often content themselves with picking out illustrative examples without systematically characterising the material as a whole. Interview material is nearly always complex enough to afford "illustrations" of whatever notion an author might choose to propose. At worst, the material can be virtually turned inside out, read "as the Devil cites Scripture". Moreover, it is nearly impossible for any third party to check the basis of the conclu- sions drawn. Discourse analysis is a label frequently used to describe analyses of texts and documents; the ingredients, the procedures followed, are seldom specified to any greater extent.

\section{Lack of Depth}

Obviously, some methods are far more popular than others. Nonetheless, we in our field make use of a rich and varied arsenal of tools. These include surveys, quantitative content analysis, individual interviews, focus groups, participant observation, discourse analysis, rhetorical analysis, semiotic analysis, etc., etc.

Experimental methods are also experiencing something of a revival after many years out in the cold. At the same time, ever fewer studies can claim a foundation in a profound and reflective methodological awareness. This is true, not least, of field studies of various sorts. A study may, for example, be said to be inspired by ethnographic methods without any further discussion of what that might mean. Reports of interview-based studies say nothing about the kinds of questions put to respondents or the techniques used to probe, to follow up informants initial responses in order to elicit more information. One reason for not including as much as an overview of the techniques used may be that the author simply does not know enough about interview technique, but has proceeded on the basis of his or her everyday social competence, combined with a number of "practice runs". Group interviews may be conducted in a variety of ways, and the interviewer or moderator can assume a variety of roles, but the choices made in these regards are seldom cause for reflection.

Multimethodological approaches within the framework of a single project have become increasingly popular as a means to broaden the perspective on the phenomenon at hand. Such an approach presumes, however, competence in a broader spectrum of methods than studies applying a single method do. No matter how common the practice may have become, it is not sufficient to state that the findings have been confirmed by triangulation, using the methods applied, and then simply list them: say, discourse analysis, interviews and observation. Integration of different methods requires a thorough familiarity with the methods involved. Today I see a real risk that we are well on our way toward a superficial eclecticism, a situation where researchers with underdeveloped methodological knowledge borrow a little here, a little there and justify it all with the word of honour triangulation. 
That training in methodology is offered in the form of smorgasbord courses offering tasty bits of this and that, but no depth, obviously has some problematic consequences. The same goes for many methods textbooks. There are today, in both the English and the Swedish literature, any number of elementary textbooks on methods, whereas titles offering a thorough grasp of any one method are scarce. As a consequence, the relation to methodology imparted to new generations of researchers have become non-reflective and superficial. Methodological issues are not taken seriously, and researchers feel no compunctions about using new and diverse methods without knowing enough about them. This seem to be an age of plucky amateurism when it comes to methods.

\section{The Epistemological and Ontological Basis are Concealed}

That different research and knowledge interests are related to different methods - for example, a choice between quantitative and qualitative methods - is clear to all. But, that methods themselves are based on fundamental notions about the nature of the reality to be studied is seldom brought to light. In a spirit of pluralism and multidisciplinarity we borrow methods and perspectives from each other or combine methods without stopping to ask whether or not they spring out of compatible epistemological and ontological soil. How, for example, are we to combine a statistical survey with an ethnographically inspired qualitative study? The former is based on the assumption of a normal distribution of characteristics among the population, whereas the latter presumes the informant to be representative of the culture or subculture. How can we combine qualitative with quantitative content analysis? A textual analysis in the Arts tradition may be based on implicit structuralist assumptions, or the opposite, an assumption that the object is unique. A social science-inspired analysis will instead presume a variation in the material that can be handled by means of systematic sampling and statistical analysis. Patterns are revealed through analysis of extensive material. By what rationale can the the two standpoints be integrated?

The discussion of ontological assumptions will differ to some extent, depending on whether we are dealing with textual analysis (in the sense of documentary material like newspaper articles, television programmes, films or documents) or we are dealing with analysis of human material (individuals, groups or institutions). Our interest lies not in the human body, clothing, or what places of work look like, but rather phenomena like attitudes, values, behaviour and meaning-producing processes, communication and interaction. We have to pose questions via postal questionnaires or interviews, engage in participant observation, etc., in order to get a material.

In the case of analysis of "human material", the researcher first creates his/her material and then analyzes what he/she has constructed. It is by all means an exacting task. We find to some extent a similar constructivist element in textual analysis inasmuch as the researcher selects the material to be analysed and the perspectives from which he/she approaches it. Newspapers and television programmes exist objectively, however, quite independent of the researcher; the researcher is not the producer of the texts he or she studies.

It is important that we ask ourselves: What do we construct, and what can we construct? It is necessary for us to relate to different levels and different theoretical perspectives. Take, for example, constructivism - in vogue today and often taken as an epistemological standpoint: knowledge is not discovered, it is constructed. That is all well and good, as far as it goes, but there are also some implicit ontological positions. Radical constructivists hold that we can only construct specific, situation-bound speech, an ontological position that might be formulated as: "When people talk to each other, the world gets constructed" (Burr, 1995: 7). In this view, there is no meaning beyond the temporal and spatial extent of the conversation. This position is embedded in quite a number of media studies and professed by researchers who favour focus groups and conversation analysis. I do not agree with the radical constructivist position; personally, I am an adherent of a more moderate strain. But that it not the point. What I am trying to say is that we need to make the implicit ontological assumptions on which various schools rest, and their methodological consequences, explicit.

Another question of an ontological nature is: Who does the informant or respondent represent? The assumption underlying traditional surveys is that characteristics vary and are distributed throughout the population, or parts of it, in a normal distribution. Culture is conceived of as being heterogeneous, and randomized samples guarantee that the results of one,s survey will be representative and generalizable. The respondent represents him/herself, and whoever else may resemble him/her is an empirical question. Ethnological studies rather conceive of individuals as bearers of their entire cul- 
ture, and the informant - only a few are generally required - represents the culture or the particular subculture to which he/she belongs. Here the researcher may choose informants who are knowledgeable and able to speak at length on cultural values, behaviours, habits, etc. Some psychological studies are based on an even higher degree of implicit generalization, namely, an ontology that conceives of the informant/individual as a representative of humanity. Essentially human processes are presumed to exist, and they are the focus of the researcher, s quest. Laboratory experiments - to investigate attention or memory processes, for example - are preferred as they afford a high degree of control. Students also constitute an abundant supply of experimental subjects. Piaget's theory of development in young children is based on studies of his own child. That is entirely possible, given a universal ontology.

A corresponding question applies to pure text analyses: Who does the text represent? A structuralist text analysis takes its point of departure in assumptions about essential structures that in principle are present in all texts, or at least in all texts in a given genre. These assumptions lead to entirely different ideas about the choice of texts to be included in the analysis - a single text may be sufficient than, for example, those that apply to a quantitative content analysis. The latter is involved with basic assumptions about variation, which must be approached via systematic samples.

On a superficial level we may have different opinions about the merits of the different schools, but a fruitful dialogue will not occur until we bring these implicit and different ontological starting points out into the open.

\section{The Methodological Problems of Multidisciplinarity}

A multidisciplinary field like media and communication studies is always at risk of succumbing to superficial eclecticism and a lack of both stringency and depth as regards methodological sensibilities. We borrow at will without bothering to acquire a command of the methodological competence each discipline has developed over longer periods of time. We mix methods without stopping to ponder the epistemological and ontological prerequisites for their successful integration. The training we offer our students consists of smorgasbord courses, in which we in rapid succession try to give a basic orientation to a series of methods, all the while knowing that each method demands considerable depth and insight. The same goes for the literature on methodology. Interview techniques occupy a single chapter, whereas there are dozens of whole books devoted to the interview as method, just waiting to be read.

Of course, we are hardly the only ones experiencing these problems. Conditions at universities and in science more generally are in flux, and the changes influence how people look at methods and methodologies.

\section{The Future of Science in a Societal Perspective}

Gibbons et al. (1994) argue that science is confronted with some fundamental changes as a consequence of the massification of higher education, and the politization and commercialization of science. Traditionally, research was characterized of homogeneity and well-defined disciplines, by internal quality control systems (e.g., intradisciplinary review boards and peer review); research questions were for the most part both formulated and answered within one and the same discipline. By contrast late-modern science is characterized by heterogeneity and multidisciplinarity and the differentiation of research areas rather than disciplines. Multiple and diverse criteria of quality, some of them external - like social, economic or political utility are now at play, and research questions are often formulated by practitioners, by individuals and institutions outside the academy. Utility to society and industry is important.

We in the field of media and communication research surely find all this familiar; surely, it feels as though we are taking giant steps toward Paul Lazarsfeld,s (1941) administrative research. When Nowotny, Scott \& Gibbons (2001) contend that "research" has become more important than "science", we know what they are talking about. Science is about more than generating new knowledge; also important is the systematization of existing knowledge and making it available to coming generations of scholars and others. These ideas seem somehow tradition-bound or old-fashioned these days. Instead, the focus is on research. Research is hailed as a motor force in the economy, as a tool to promote innovative growth. "Research" has a seductive quality in the ears of politicians and others in power.

It is in this new setting that we need to develop new ways to strengthen our competence regarding methods and methodologies. Both the social sciences and humanities are characterized by transcendence and pluralism. The positivistic ideals of 
knowledge, according to which the researcher often was a specialist with profound knowledge of a very narrow subject, which he or she systematically penetrated, has been upstaged by a broader focus of knowledge interests and multiple methodological approaches. Social studies and Arts approaches are frequently combined in the same research project. Ad hoc and highly multidisciplinary research teams are another current trend (Nowotny et al. 2001).

I myself am part of a milieu, MTM (Man-Technology-Environment), that involves researchers in the natural sciences, social sciences and the Arts, namely, at Örebro University. Such milieus certainly can be very stimulating, but also extremely frustrating with many and contradictory methodological demands that can be quite confusing, especially for the doctoral students involved in them.

Research is no longer the province of a chosen few. The field has broadened immensely in terms of both the number of post-graduates and the number of institutions engaged in research. The massification of higher education has stimulated the production of a great many "introductions to methods and methodology" but has also led to a relative dearth of literature that offers an in-depth grasp. It has also made it difficult to maintain an even (and high) level of methodological training.

Other forces for change are heightened demands regarding the pace of research. There is less willingness to wait for findings and publications and stronger pressure to satisfy the knowledge needs of external actors. In Sweden the signals could hardly be clearer: "Commercialize the results of research," urged Minister of Education Thomas Östros in Dagens Nyheter 5th February 2004, (after a few customary honorific obeisances); "Let industry's needs steer research," urged Swedish labour leader Wanja Lundby-Wedin and representatives of Swedish industry and research councils in the same paper only a few weeks later (11th April).

According to Nowotny et al. (2001), rather than scientific knowledge, what is in demand these days is data. The shift in focus threatens to marginalize methodological concerns, not least concerns about validity. An illustrative example of this kind of shift is the commercialization of audience research in broadcasting, the aduience ratings. Television viewing is now measured 'round the clock' by means of electronic monitoring devices, "people meters". The interest in instant rating figures is motivated not so much by an interest in the phenomena of television viewing as the need for a measure of success that will impress advertisers and advertising brokers. The electronic devices record when the television set is switched on and to what channel. No deeper analysis of this horde of numbers is performed.

The ongoing ideological struggle concerning who should formulate questions for research and how research should be conducted is, of course, hardly specifically Swedish. The same forces are at play all over Europe.

\section{Conclusion}

The questions to be addressed and answered should guide the choice of research method or methods. We have no reason to revert back to the kind of methodological fundamentalism that measured the "quality" of science according to whether or not a prescribed method was used, or to a situation where the method dictated the research questions posed. Transcendent and multidisciplinary approaches can result in broader knowledge and deeper insights about phenomena in reality, which, after all, always are at once cultural, social and human. But we have every reason to pay more attention, and put more into methodological training with respect to both the theoretical bases for one, s choice of methods and the skills required to apply the methods chosen.

In sum, then, several factors that have tended to weaken interest in knowledge of methods - in our field as well as in others - have been identified:

- multidisciplinary transcendence of the bounds of traditional disciplines on the surface, but without integration at deeper levels;

- the mass production of introductory textbooks on methods and methodology, but relatively little thoroughgoing literature;

- a shortage of time and little reflection in researchers' day-to-day working lives;

- a higher rate of through-put at both postgraduate and undergraduate levels;

- commercialization of research with greater deference to the needs and interests of economic players of various kinds. 


\section{References}

Burr, V. (1995) An Introduction to Social Constructionism. London: Routledge.

Fetveit, A. (2000) Den trojanske hest: Om metodebegrepets marginalisering av humanistisk medieforskning [The Trojan horse: On the marginalization of method in research in the Humanities]. Norsk Medietidskrift, 2: $5-27$.
Gibbons, M., Limoges, C., Nowotny, H., Schwartzman, S., Scott, P. and M. Trow (1994) The New Production of Knowledge. The Dynamics of Science and Research in Contemporary Societies. London: Sage.

Lazarsfeld, Paul F. (1941) Administrative and Critical Communication Research. In Lazarsfeld (ed.) Qualitative Analysis. Historical and Critical Essays. Boston: Allyn and Bacon, Inc. 1972, Ch. 6.

Nowotny, H., Scott, P. and M. Gibbons (2001). Re-Thinking Science. Knowledge and the Public in an Age of Uncertainty. Cambridge, UK: Polity Press 